\title{
Similarity in the difference: changes in community functional features along natural and anthropogenic stress gradients
}

\author{
Cayetano Gutiérrez-Cánovas, ${ }^{1,4}$ David Sánchez-Fernández, ${ }^{2}$ Josefa Velasco, ${ }^{1}$ Andrés Millán, ${ }^{1}$ \\ AND NÚRIA BONADA ${ }^{3}$ \\ ${ }^{1}$ Departamento de Ecología e Hidrología, Universidad de Murcia, Campus de Excelencia Internacional Regional \\ "Campus Mare Nostrum”, 30100 Murcia, Spain \\ ${ }^{2}$ Institut de Biologia Evolutiva (CSIC-UPF), Passeig Maritim de la Barceloneta 37-49, 08003 Barcelona, Spain \\ ${ }^{3}$ Grup de Recerca Freshwater Ecology and Management (FEM), Department d'Ecologia, Facultat de Biologia, \\ Universitat de Barcelona, Diagonal 643, 08028 Barcelona, Spain
}

\begin{abstract}
The effect of stressors on biodiversity can vary in relation to the degree to which biological communities have adapted over evolutionary time. We compared the responses of functional features of stream insect communities along chronic stress gradients with contrasting time persistence. Water salinity and land use intensification were used as examples of natural (long-term persistent) and anthropogenic (short-term persistent) stressors, respectively. A new trait-based approach was applied to quantify functional diversity components and functional redundancy within the same multidimensional space, using metrics at the taxon and community levels. We found similar functional responses along natural and anthropogenic stress gradients. In both cases, the mean taxon functional richness and functional similarity between taxa increased with stress, whereas community functional richness and functional redundancy decreased. Despite the differences in evolutionary persistence, both chronic stressors act as strong nonrandom environmental filters, producing convergent functional responses. These results can improve our ability to predict functional effects of novel stressors at ecological and evolutionary scales.
\end{abstract}

Key words: anthropogenic land use; functional niche; global change; habitat filtering; natural stress; predictive ecology; stream insects; water salinity.

\section{INTRODUCTION}

Predicting ecosystem responses to environmental change is one of the most challenging tasks that ecologists face. The effects of stressors on biodiversity can vary in relation to the degree to which biological communities have adapted over evolutionary time (Buchwalter et al. 2008, Garbuz et al. 2008, GutiérrezCánovas et al. 2013). Although stress may trigger microevolutionary responses even acting at the short term (millennia to decades, as reported by Bradshaw and Holzapfel 2006, Cothran et al. 2013, Breckels and Neff 2014, Higgins et al. 2014), long-term persistence of stressors plays a crucial role in speciation (Parsons 2005) and stress tolerance acquisition (Jump and Peñuelas 2005, Buchwalter et al. 2008, Garbuz et al. 2008). Therefore, the adaptation to stress could be considered as a continuum over time, representing anthropogenic $(<10000 \mathrm{yr})$ and natural stressors (long-term persistent) as different steps along such an axis.

Ecological theory predicts nonrandom species sorting in association with environmental filters, determined by species traits (Southwood 1977, Shipley et al. 2006,

Manuscript received 30 July 2014; revised 2 February 2015; accepted 9 February 2015; final version received 5 March 2015. Corresponding Editor: E. Van Donk.

${ }^{4}$ E-mail: cayeguti@um.es
Weiher et al. 2011, Mouillot et al. 2013). As functional features (i.e., within-taxon trait mean and variability) conferring stress tolerance arise in response to highly selective environmental filters (Buchwalter et al. 2008, Rodrigues et al. 2013), it is likely that some of the functional attributes of communities subjected to natural, long-term persistent stressors may be similar to those related to relatively new, short-term anthropogenic stressors. Furthermore, previous studies have documented that traits conferring natural stress tolerance can also grant a species the ability to tolerate novel stressors (Badyaev 2005, Buchwalter et al. 2008, Arribas et al. 2014). In both cases, a subset of biological features will be shared among the different organisms that inhabit naturally and anthropogenically stressed environments (Southwood 1977, Odum 1985, Stanton et al. 2000). These commonalities should result in similar trends of functional features along stress gradients, irrespective of the degree to which a species has adapted and specialized over evolutionary time (i.e., to a natural or anthropogenic stressor). In this case, stress would act as a strong nonrandom environmental filter that configures trait evolution and community functional properties. Otherwise, the functional features of naturally and anthropogenically stressed ecosystems would show clear divergences, reflecting a differential filtering process during colonization and adaptation. 
Functional measures at the community level are related to ecosystem functioning and stability (Hooper et al. 2005) and are able to reflect nonrandom community assembly processes (Villéger et al. 2008). For example, whole-community functional diversity measures, such as functional richness and divergence, or functional redundancy, are predicted to decrease with increasing environmental filtering (Weiher et al. 2011, Mouillot et al. 2013). However, these measures are generally estimated using mean trait values for each taxon, despite the fact that some studies have demonstrated the importance of within-taxon trait variability to better explain community assembly (Stubbs and Wilson 2004, Violle et al. 2012). Therefore, the advantages of using within-taxon functional measures are twofold: (1) improving whole-community measures, and (2) allowing the estimation of new functional measures at the taxon level. As an example of the first case, within-taxon trait information provides a direct measure of community functional overlap among taxa (i.e., functional redundancy), eliminating the need to classify species into functional groups, which may result in some loss of information (Villéger et al. 2008). Regarding the second case, taxon functional richness can be estimated as the functional space filled by the variability exhibited by each taxon (Stubbs and Wilson 2004). Taxa showing higher functional richness are more likely to be present in a wider range of environmental conditions than more functionally homogeneous taxa, which are thought to be more sensitive to environmental change (Scoville and Pfrender 2010, Latta et al. 2012). Functional similarity between taxa is an additional within-taxon measure, which indicates the mean relative overlap between pairs of taxa in a community, thereby allowing detection of community assembly rules in relation to mean trait similarity. For example, limited similarity of coexisting species due to interspecific competition prevails under non-stressful conditions (MacArthur and Levins 1967, Stubbs and Wilson 2004), whereas intense habitat filtering increases functional similarity between taxa (Weiher et al. 2011, Maire et al. 2012).

Here, we explored changes in community functional features along chronic stress gradients with contrasting time persistence. In particular, we compared changes in stream insect communities along a water salinity gradient (i.e., as a natural, long-term persistent stress) and across different land uses (i.e., as an anthropogenic, short-term persistent stress) as stress gradients, using within-taxon functional measures at the taxon (mean taxon functional richness and mean functional similarity between taxa) and whole-community (community functional richness, functional dispersion, and functional redundancy) levels. We selected streams as focal ecosystems because they are (1) well-defined and stable habitats over geological timescales, allowing long-term speciation (e.g., Arribas et al. 2014); and (2) characterized by marked environmental gradients in relation to which taxonomic and functional responses are detected (e.g., Díaz et al. 2008, Gutiérrez-Cánovas et al. 2013).

\section{Methods}

To study the functional responses of aquatic insects to natural and anthropogenic stresses, we chose one example of each type. As a natural stressor, we selected water salinity due to (1) its persistence over evolutionary time scales in the study region (Arribas et al. 2014), and because (2) the relationships between salinity and the taxonomic and functional attributes of aquatic fauna are well known (Díaz et al. 2008, Millán et al. 2011, Arribas et al. 2014). As an anthropogenic stressor, we chose land use intensification because (1) it is one of the most important stressors that affects aquatic ecosystems worldwide (Sala et al. 2000, Cooper et al. 2013), and (2) it is closely associated with increasing human populations (Scanlon et al. 2007). Because stressors showing high temporal variability may yield complex ecosystem responses (Gutiérrez-Cánovas et al. 2013), we only selected chronic environmental stressors, which act as perennial habitat filters for the regional species pool.

We focused our study on western Mediterranean streams (Iberian Peninsula and Italy, see Appendix A: Fig. A1), gathering data from published work, gray literature, and our own resources (see Appendix A for further details). We selected this study area because (1) the regional pool of aquatic taxa is well known in terms of taxonomy, biological traits, and ecological responses (Díaz et al. 2008, Bonada and Dolédec 2011, Millán et al. 2011); and (2) it presents the natural (water salinity) and anthropogenic (land use intensification) stressors examined in this study (Bonada and Resh 2013).

\section{Environmental data sets}

The salinity data set included unpolluted (i.e., drained catchments mainly covered with natural or semi-natural vegetation or nonirrigated crops), small (width $<10 \mathrm{~m}$ ), mid-mountain and lowland streams $(<1200 \mathrm{~m}$ above sea level) with a permanent flow (see Appendix A); these criteria resulted in a total of 105 sites. Some of these streams drain evaporitic catchments resulting in naturally highly mineralized waters (Millán et al. 2011). The salinity gradient ranged from electrical conductivities of $100 \mu \mathrm{S} / \mathrm{cm}$ to near $300000 \mu \mathrm{S} / \mathrm{cm}$. We used the electrical conductivity of water as an indicator of osmotic stress.

The land use data set included perennial, freshwater (conductivity $<2500 \mu \mathrm{S} / \mathrm{cm}$ ), small and intermediate (width $<40 \mathrm{~m}$ ), mid-mountain and lowland streams ( $<1200 \mathrm{~m}$ above sea level; see Appendix A), resulting in a total of 102 sites. This data set contained streams that drain from natural vegetation catchments to highly modified catchments. We measured anthropogenic land use intensity as the sum of the percentage areas of crops under irrigation and urban land in the catchment $5 \mathrm{~km}$ upstream of each sampling point (i.e., the intersection between the entire catchment and a $5 \mathrm{~km}$ radius buffer centered on the sampling point). These two land uses 
have been associated with nutrient enrichment and habitat degradation at the scale we studied (Monteagudo et al. 2012). These effects may not be completely new for the regional species pool, since nutrient enrichment usually produces an increase in salinity. However, despite the fact that these two stress gradients may have overlapping effects at conductivities $<2500 \mu \mathrm{S} / \mathrm{cm}$, they affect organisms through increasing different physical and chemical mechanisms as both stresses intensify (Díaz et al. 2008). Land uses were obtained from the most up-to-date land use map that predated the time when the invertebrate sample was obtained (Corine Land Cover 1990, 2000, and 2006 layers). ${ }^{5}$

\section{Biological data sets}

We obtained community composition data from the sites where water salinity (105 sites) or land use data were available (102 sites), as indicated above. At each site, the taxonomic composition was obtained by pooling up to four samples that corresponded to different seasons. This approach is adequate to represent the mean annual community, even when samples are collected from only a single season (Bonada et al. 2007). We focused our analysis on genera belonging to the main insect orders present in aquatic habitats (i.e., Coleoptera, Ephemeroptera, Hemiptera, Odonata, Plecoptera, and Trichoptera), as genus-level information is sufficiently accurate to study the functional diversity of rivers (Dolédec et al. 2000, Gayraud et al. 2003). We excluded dipterans due to the paucity of detailed trait information on these species and their complex taxonomy (Pfenninger et al. 2007). The groups of insects considered in this analysis show contrasting ecological responses to stress that are associated with different biological traits (Harrison and Dobson 2008), some of which are good surrogates for macroinvertebrate biodiversity in aquatic habitats (Sánchez-Fernández et al. 2006, Dijkstra et al. 2014).

To characterize the functional properties of the communities, we used a database containing information on 13 biological traits at the genus level (e.g., morphology, life history, aquatic stage, dispersal, resistance forms, respiration, locomotion, and feeding habits; see Appendix B). Eleven out of these 13 biological traits were obtained from Tachet et al. (2002). The other two traits (i.e., body form and the degree of body sclerotization; see Appendix C), were codified for this study based on taxonomic expertise and published taxonomic keys (Tachet et al. 2002). Each genus was coded according to the degree of affinity of each species with each trait category. To do this, we considered all biological information available within organisms belonging to each genus, using a fuzzy coding approach (Chevenet et al. 1994). Before analyzing data, fuzzy coding data were converted to percentages of

${ }^{5}$ http://www.eea.europa.eu/data-and-maps/ affinity for each trait. This procedure standardizes the potential differences in the codification scores (i.e., different row sums for each taxon and trait). More details on the environmental and biological data sets used here can be found in Statzner et al. (2007).

\section{Data analysis}

We employed the following three matrix types to characterize the functional space: a presence/absence taxonomic matrix (hereafter, sites $\times$ taxa); a matrix containing the fuzzy coded trait profile for each taxon (hereafter, taxa $\times$ traits); and the resulting communitylevel trait matrix (hereafter, sites $\times$ traits) that represents the mean trait profile for each site.

We defined the functional space as a multidimensional Euclidean space where the axes summarized the variability of a subset of relevant traits that responded to both stressors, assuming that some trait features may be necessary to establish and maintain viable populations in stressful habitats. To define this space for each type of stressor, we calculated the Pearson correlation coefficients between trait categories and the stress intensity for each data set separately using the sites $\times$ traits matrices. We retained the minimum set of traits with an average absolute coefficient of $R \geq|0.30|$ for both data sets (see Appendices B and C). Trait selection is necessary in all types of analyses to illustrate functional patterns clearly (Villéger et al. 2008). Our aim was to use the same suite of traits to explore the response of functional features to both environmental gradients. Although this suite of traits might be different if only one type of stressor is considered, it is useful to illustrate how similar functional patterns would emerge in response to chronic stress when considering common relevant traits. The retained set included the number of generations per year, body form, and degree of sclerotization, to characterize functional niches (see Appendix C). The number of generations per year is positively related to resilience to disturbance (Southwood 1977). Body form and the degree of sclerotization play a fundamental role in dispersion, as fully sclerotized and robust adults are able to avoid desiccation during flight (Harrison and Dobson 2008). Body form is also related to hydraulic stress (Lamouroux et al. 2004), and the degree of sclerotization is related to resistance against physical and chemical impacts (Hopkins and Kramer 1992).

We developed different $\mathrm{R}$ functions ( $\mathrm{R}$ Development Core Team 2013) to estimate five functional measures based on within-taxon trait information to account for the changes in the functional space at the taxon and at the whole-community levels (see Appendix D and Supplement for details). Mean taxon functional richness and mean functional similarity between taxa were calculated at the taxon level, while functional richness, functional dispersion, and functional redundancy were quantified at the whole-community level. Mean taxon functional richness measures the mean capacity of the 
taxa belonging to a community to fill the functional space. It ranges from zero in the absence of taxa to higher values when the community is composed of functionally variable taxa that fill a high extent of the functional space. Mean functional similarity between taxa represents the relative overlap between taxon pairs. It goes from zero when there are no taxa or when the occurring taxa are functionally distinct (no overlap) to one when all the taxa composing community are functionally identical. Community functional richness estimates the filling of the functional space. It ranges from zero when there are no taxa to higher values when organisms show highly different trait values. Community functional dispersion is a measure of the distribution of taxa in the functional space. It reaches zero in absence of taxa, presenting higher values when there are taxa with traits differing greatly from the mean community values. Finally, community functional redundancy quantifies the degree of niche overlap in the functional space. This variable is zero in the absence of taxa or when there is no overlap between taxon pairs, reaching higher values when there are many functionally similar and diverse taxa.

The relationship between these functional measures and stressor gradients was tested using generalized linear models (GLMs), assuming a Gaussian distribution of the dependent variables (see the Supplement). We only evaluated linear relationships between dependent and independent variables to test our initial predictions. For the mean functional similarity between taxa, we selected sites with a taxonomic richness $>1$ to avoid potential bias, as the mean functional similarity $=1$ when taxonomic richness $=1$. The mean functional similarity between taxa and functional redundancy were logtransformed to improve linearity with stressors. The predictors were $z$-standardized (mean $=0$ and standard deviation $=1$ ) after appropriate transformations (logtransformation for conductivity and arcsine square-root transformation for the percentage of land use intensity).

\section{Null models}

A relationship between stress and functional variables can be found simply as a result of an underlying taxonomic richness gradient (Villéger et al. 2008, 2011). This uncertainty is due to the probability of finding more or fewer trait categories solely as a consequence of the number of taxa (i.e., sampling effect). To assess the nonrandomness of the observed trends, empirical parameters should be distinct from those resulting from relating the stress intensity to functional variables obtained after randomizing communities with the same richness gradient and taxon frequency of occurrence but a random composition. To perform this assessment, we assembled 999 artificial communities in which taxa were drawn randomly from the taxonomic pool for each data set with fixed row (taxon richness) and column (taxon frequencies) totals (Ulrich and Gotelli 2007). For each simulation, we calculated the mean taxon functional richness, mean functional similarity between taxa, community functional richness, community functional dispersion, and community functional redundancy, and re-examined their relationship with the putative stressor to obtain the intercept and slope for each relationship (i.e., using the same procedure as for the empirical data). We examined the null model's statistical significance using an exact two-tailed test to estimate the probability that the observed value would be significantly $(\alpha=0.05)$ greater or smaller than the null distribution.

All statistical analyses were performed in $\mathrm{R}$ (ade4, plyr, and vegan libraries; R Development Core Team 2013).

\section{Results}

The models relating functional features to natural and anthropogenic stressors were all significant and showed similar patterns, regardless of the type of stressor (Table 1 and Fig. 1). In general, more deviance was explained in the salinity data set models $(16.1-71.6 \%)$ than in the land use data set models (12.8-40.7\%). The functional features that were best explained by stress were community functional richness (salinity, 71.6\%; land use: $36.4 \%$ ) and functional dispersion (salinity, 69.1\%; land use, $40.7 \%$ ), followed by the mean functional similarity between taxa (salinity, 65.1\%; land use, $19.0 \%$ ). Stress explained less of the deviance in the mean taxon functional richness (salinity, 35.9\%; land use, $12.8 \%$ ) and community functional redundancy (salinity, 16.1\%; land use, 22.9\%).

Functional features showed similar trends in response to natural and anthropogenic stressors (Fig. 1, Table 1). The mean taxon functional richness and mean functional similarity between taxa (both measured at the taxon level) showed positive slopes in relation to stress intensity for the salinity and land use data sets. In contrast, the three features measured at the wholecommunity level (i.e., community functional richness, functional dispersion, and functional redundancy) showed negative slopes in relation to stress intensity in both data sets (Fig. 1).

The empirical model parameters were generally distinct from those obtained from models relating simulated functional attributes to stressors, reflecting nonrandom changes along natural and anthropogenic stress gradients (Table 2); of the intercepts and slopes for the empirical models, 9 out of 10 were significantly distinct from the null distributions. For the salinity data set, the intercept of the mean taxon functional richness models was indistinguishable from the null distribution $(z$ score $=1.32 ; P=0.105)$, whereas for the land use data set, the slope of the functional dispersion ( $z$ score $=$ $-1.41 ; P=0.087$ ) model was not significantly different from the null distribution. For both data sets, the empirical slopes showed significantly higher values for the mean taxon functional richness (salinity, $z$ score $=$ 4.04, $P=0.001$; land use, $z$ score $=3.37, P=0.001$ ), mean functional similarity between taxa (salinity, $z$ score 
TABLE 1. Results of generalized linear models relating the response of community functional features to natural (i.e., salinity) and anthropogenic (i.e., land use) stressors.

\begin{tabular}{lccc}
\hline \hline \multicolumn{1}{c}{ Data set } & Model & $P$ & $\begin{array}{c}\text { Explained } \\
\text { deviance }\end{array}$ \\
\hline Mean taxon functional richness & & & \\
$\quad$ Salinity & $y=3.13+0.47 x$ & $<0.001$ & 35.9 \\
$\quad$ Land use & $y=3.01+0.30 x$ & $<0.001$ & 12.8 \\
Mean functional similarity between taxa & & & \\
$\quad$ Salinity & $y=-1.53+0.35 x$ & $<0.001$ & 65.1 \\
$\quad$ Land use & $y=-1.59+0.16 x$ & $<0.001$ & 19.0 \\
Community functional richness & & & \\
$\quad$ Salinity & $y=15.06-3.14 x$ & $<0.001$ & 71.6 \\
$\quad$ Land use & $y=15.69-2.14 x$ & $<0.001$ & 36.4 \\
$\quad$ Functional dispersion & & & \\
$\quad$ Salinity & $y=3.38-0.41 x$ & $<0.001$ & 69.1 \\
$\quad$ Land use & $y=3.50-0.27 x$ & $<0.001$ & 40.7 \\
Functional redundancy & & & \\
$\quad$ Salinity & & & \\
$\quad$ Land use & $y=5.47-0.52 x$ & $<0.001$ & 16.1 \\
\hline
\end{tabular}

Notes: The significance of the linear coefficients $(P)$ and the percentage of explained deviance are also shown for each model. Salinity data set, $n=105$; land use data set: $n=102$.

$=7.02, P=0.001 ;$ land use, $z$ score $=2.48, P=0.006)$, and community functional redundancy (salinity, $z$ score $=8.54, P=0.001 ;$ land use, $z$ score $=1.75, P=0.049$ ), while the slope of the empirical models relating community functional richness to stress showed significantly lower values (salinity, $z$ score $=-6.42, P=0.001$; land use, $z$ score $=-1.72, P=0.048$ ).

\section{DisCussion}

The functional properties of communities responded similarly along natural and anthropogenic gradients of stress. These changes included a greater mean taxon functional richness and mean functional similarity between taxa, and a lower community functional richness and functional redundancy associated with increased stress. These results highlight the importance of deterministic processes of trait filtering during the different stages of colonization and the response and adaptation to chronic stress over time. The similarity of the responses observed here indicates that the two types of stress constrain functional features of organisms and communities in an analogous manner, regardless of the evolutionary adaptations acquired over geological time, and stress would therefore act as a template that configures trait evolution and community functional properties.

At the taxon level, we found that both types of stress progressively selected taxa showing more trait variability (higher mean taxon functional richness) and more homogeneous functional traits (higher mean functional similarity between taxa). Previous studies have found that trait variability can facilitate adaptation to novel or stressful environments, enhancing ecological tolerance (Badyaev 2005, Scoville and Pfrender 2010, Latta et al. 2012). In fact, adaptation to stressful habitats could arise from ecological generalization (Latta et al. 2012), where colonizers would benefit from low predation pressure and low interspecific competition. Similarly, we found higher mean functional similarity between taxa as stress increased. For each trait, habitat filtering reduces overdispersion, producing a convergence toward the optimum value (Shipley et al. 2006, Weiher et al. 2011). Accordingly, recent studies have found that stress causes functional homogenization of plants (e.g., Maire et al. 2012), fish (e.g., Pool and Olden 2012), and birds (e.g., Luck and Smallbone 2011).

We also found similar trends for functional measures estimated at the whole-community level (community functional richness and functional redundancy) in response to natural and anthropogenic stressors. This result indicates that stress is a factor that constrains community assembly similarly during the colonization process and over evolutionary time. In benign environments, assembly rules shift over evolutionary time as evolution enhances functional diversification, progressively filling the functional space (Villéger et al. 2011). In our study, the stress intensity reduced the range of the functional space filled by the community, due to nonrandom environmental filtering. This process sorted the species located in certain parts of the functional space, particularly those occurring at extreme positions along the axes. However, for community functional dispersion, we found that the slope of the land use data set was indistinguishable from those resulting from null models. Previous studies have reported both random and nonrandom reductions in functional richness with increased anthropogenic stress. For example, Flynn et al. (2009) detected nonrandom changes in functional richness with land use intensification for animals but not for plants.

Our results also showed that stress diminished community functional redundancy to a lesser extent 

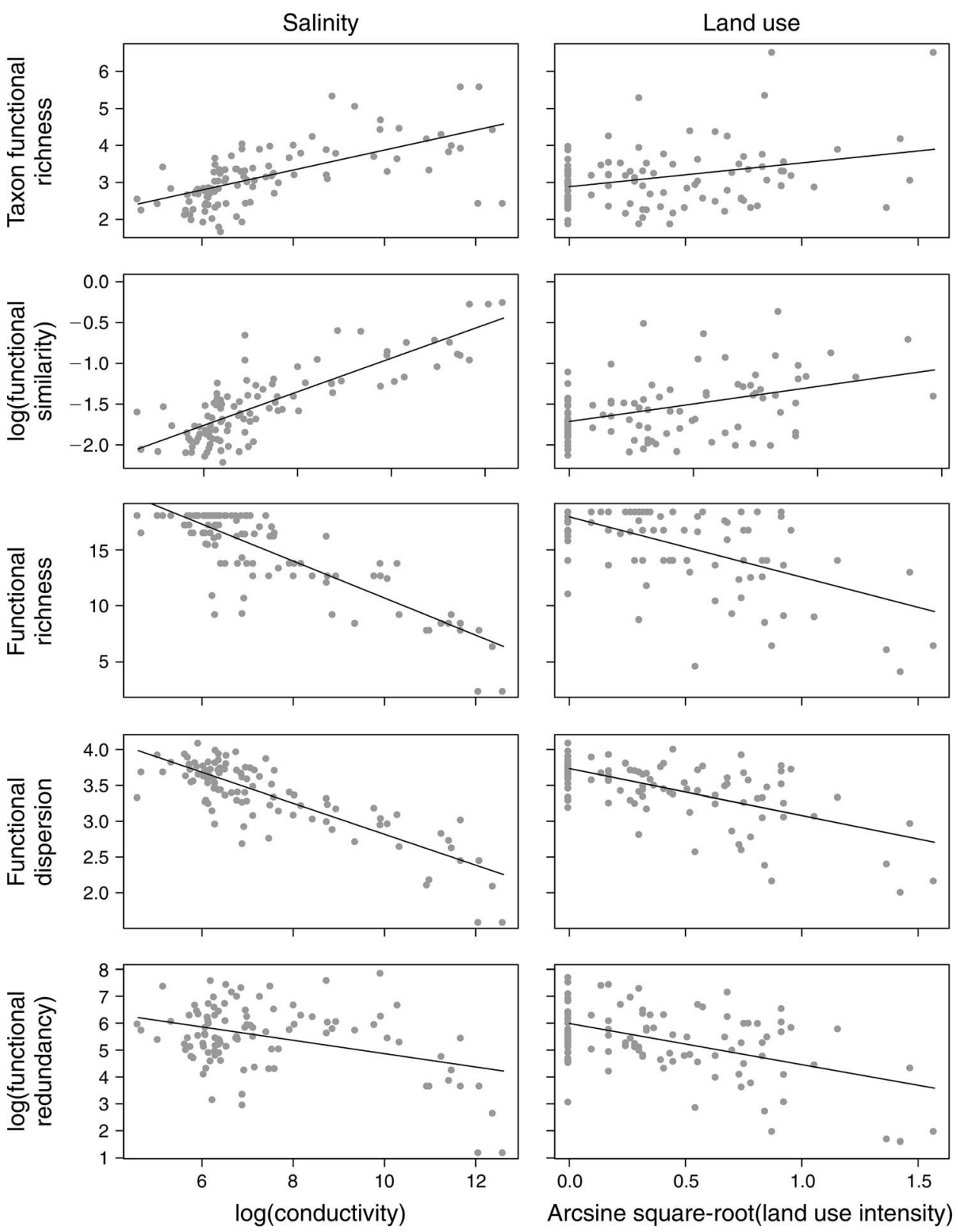

FIG. 1. Plots showing the responses of functional features to natural (i.e., salinity) and anthropogenic (i.e., land use) stressors. Solid lines represent the fitted values of generalized linear models (see Table 1 for model details). Salinity data set, $n=105$; land use data set, $n=102$

than expected by chance, revealing nonrandom filtering of taxa with traits placed in the most sensitive regions of the functional space and producing a net reduction in niche overlap among taxa, while the remaining species were progressively more similar (i.e., mean functional similarity between taxa increased). Other studies have found a decrease in functional redundancy along anthropogenic stress gradients for plants (e.g., Laliberté et al. 2010), soil microbes and invertebrates (e.g., Salminen et al. 2001), and aquatic invertebrates (e.g., Schmera et al. 2012). Species are able to colonize and adapt to less stressful habitats through trait diversification and speciation, thus increasing functional redundancy over evolutionary time as a result of increasing both the number of species and functional identities (Villéger et al. 2011). Conversely, the similarity of the response to long-term and anthropogenic persistent stressors supports the idea that habitat filtering constrains trait evolution in organisms residing in stressful habitats, resulting in high inter-taxon similarity, as observed here, but reducing the chances for species to colonize or adapt to these environments, 
TABLE 2. Results of two-tailed significance tests examining the differences between the empirical parameters of models that relate functional features to natural (i.e., salinity) and anthropogenic (i.e., land use) stresses and the null distribution of parameters from models relating the same stress gradients to functional features calculated from 999 artificial communities built with fixed row and column totals (see Methods).

\begin{tabular}{|c|c|c|c|c|c|c|c|c|c|c|}
\hline \multirow[b]{2}{*}{ Model } & \multicolumn{2}{|c|}{$\begin{array}{l}\text { Mean taxon } \\
\text { functional richness }\end{array}$} & \multicolumn{2}{|c|}{$\begin{array}{c}\text { Mean functional } \\
\text { similarity } \\
\text { between taxa }\end{array}$} & \multicolumn{2}{|c|}{$\begin{array}{c}\text { Community } \\
\text { functional richness }\end{array}$} & \multicolumn{2}{|c|}{$\begin{array}{l}\text { Community } \\
\text { functional } \\
\text { dispersion }\end{array}$} & \multicolumn{2}{|c|}{$\begin{array}{l}\text { Community } \\
\text { functional } \\
\text { redundancy }\end{array}$} \\
\hline & $z$ & $P$ & $z$ & $P$ & $z$ & $P$ & $z$ & $P$ & $z$ & $P$ \\
\hline \multicolumn{11}{|l|}{ Salinity } \\
\hline $\begin{array}{l}\text { Intercept } \\
\text { Slope }\end{array}$ & $\begin{array}{l}1.32 \\
4.04\end{array}$ & $\begin{array}{l}0.105 \\
0.001\end{array}$ & $\begin{array}{l}3.09 \\
7.02\end{array}$ & $\begin{array}{l}0.001 \\
0.001\end{array}$ & $\begin{array}{l}-8.20 \\
-6.42\end{array}$ & $\begin{array}{l}0.001 \\
0.001\end{array}$ & $\begin{array}{l}-5.55 \\
-4.54\end{array}$ & $\begin{array}{l}0.001 \\
0.001\end{array}$ & $\begin{array}{l}7.43 \\
8.54\end{array}$ & $\begin{array}{l}0.001 \\
0.001\end{array}$ \\
\hline \multicolumn{11}{|l|}{ Land use } \\
\hline $\begin{array}{l}\text { Intercept } \\
\text { Slope }\end{array}$ & $\begin{array}{l}3.49 \\
3.37\end{array}$ & $\begin{array}{l}0.001 \\
0.001\end{array}$ & $\begin{array}{l}2.50 \\
2.48\end{array}$ & $\begin{array}{l}0.005 \\
0.006\end{array}$ & $\begin{array}{l}-3.83 \\
-1.72\end{array}$ & $\begin{array}{l}0.003 \\
0.048\end{array}$ & $\begin{array}{l}-3.11 \\
-1.41\end{array}$ & $\begin{array}{l}0.003 \\
0.087\end{array}$ & $\begin{array}{l}2.60 \\
1.75\end{array}$ & $\begin{array}{l}0.011 \\
0.049\end{array}$ \\
\hline
\end{tabular}

Notes: The $z$ values $(z)$ and significance $(P)$ are reported.

resulting in low taxonomic richness (e.g., GutiérrezCánovas et al. 2013).

We found that multivoltinism, a sclerotized body, and flattened and cylindrical forms may allow organisms to colonize and survive in habitats subject to anthropogenic sources of stress (Southwood 1977, Buchwalter et al. 2008). There might be two complementary hypothetical explanations for this phenomenon. The first one is that some of these traits, or even other non-analyzed covariating features, have evolved recently as a result of the high selective pressure. The other hypothesis is an adaptation through exaptation (i.e., a shift in the function of one trait during evolution to cope with novel conditions) over evolutionary time (Arribas et al. 2014). In this case, the selected traits should be plesiomorphic (i.e., shared within members of the lineage) rather than apomorphic (i.e., an innovation within the lineage) because they are common in most organisms facing stresses of a different nature, including anthropogenic stressors (Southwood 1977, Herbst 2001). Recent evidence suggests that some lineages are more likely to withstand stressful conditions than expected by chance due to the presence of certain traits that enhance stress tolerance (Garbuz et al. 2008, Arribas et al. 2014). For example, among insects, many Coleoptera and Diptera species have aquatic stages adapted to a wide array of stress types. The larvae of the Chironomidae species (Diptera) are extremely ubiquitous in being able to withstand both natural (e.g., Milner et al. 2011) and anthropogenic stresses (e.g., Carew et al. 2007). There are other good examples among species of the families Stratiomyidae and Culicidae (Diptera) or Dytiscidae and Hydraenidae (Coleoptera) (Bradley 2008, Garbuz et al. 2008, Picazo et al. 2012). All these species are frequently multivoltine, and in some cases have a cylindrical, flattened or sclerotized body, as observed here. Further experiments should test the role of microevolutionary processes in the adaptation to new stressors, and if these three traits confer species tolerance to other sources of stress or even to multiple interacting stressors to allow for a more mechanistic modelling of the biological responses to environmental change.
The initial selection of traits is often critical when estimating the functional properties of communities along environmental gradients (Villéger et al. 2008), as the choice of unimportant traits can increase the probability of finding random response patterns. First, there is a probability of finding a significant functional pattern in response to environmental change simply as a result of the underlying relationship between taxonomic and functional diversities (e.g., the probability of finding rare traits in depauperate communities can be lower just by chance and not due to environmental filtering). Second, unimportant traits may obscure the functional pattern arising in response to stress, leading to a loss of explanatory power. For these reasons, we selected a suite of traits that are relevant to passing environmental filters along the studied gradients, using empirical data and mechanistic links. Additionally, we tested the nonrandomness of the relationship between functional features and stress using null models where functional features were recalculated using simulated communities with a fixed taxon number and frequency. For these reasons, we suggest that the similarity in the functional trends found here may arise from analogous assembly rules operating during colonization and over time.

Here, we demonstrated how chronic stress can be a major driver of nonrandom changes in the functional features of communities, irrespective of whether the stressor is natural (i.e., persistent over evolutionary time) or anthropogenic (i.e., short-term persistent). Our results suggest that ecosystems under intensified stress will exhibit functionally homogeneous communities composed of taxa showing wider functional niches. Functional homogenization can have consequences for ecosystem functions, particularly decreases in or a loss of stability of those functions in which stress-sensitive species are involved (Hooper et al. 2005). In addition, functionally specialized species are more likely to be lost due to stress intensification (Clavel et al. 2011, Pool and Olden 2012). The interaction between the intensification of human activities and the effects of ongoing global change will increase the number and the intensity of novel stressors that ecosystems will face in the near 
future. The approach employed here can be useful to anticipate to the functional consequences of novel stressors, contributing to making ecology a more predictive science.

\section{ACKNOWLEDGMENTS}

We thank P. Abellán, P. Arribas, O. Belmar, D. Bruno, J. A. Carbonell, S. Guareschi, S. Pallarés, and F. Picazo for their contributions in obtaining and identifying most of the macroinvertebrate samples used in this study. We are also grateful to P. Arribas, C. E. Gray, F. T. Maestre, G. Woodward, and L. Zhao for their insightful comments on earlier versions of the manuscript. We also thank the two anonymous referees for their insightful comments and suggestions. C. Gutiérrez-Cánovas was partially supported by a pre-doctoral grant from the Fundación Séneca (Agencia Regional de Ciencia y Tecnología, Región de Murcia). D. Sánchez-Fernández was supported by the "Juan de la Cierva" program of the Spanish Ministry of Economy and Competitiveness. The salinity surveys were financed by the Ministerio de Ciencia e Innovación (Spain) through $\mathrm{I}+\mathrm{D}+\mathrm{i}$ project no. CGL2006-04159/BOS (A. Millán). N. Bonada acknowledges the BioFresh EU-funded project-Biodiversity of Freshwater Ecosystems: Status, Trends, Pressures, and Conservation Priorities (7th FWP contract No 226874).

\section{Literature Cited}

Arribas, P., C. Andújar, P. Abellán, J. Velasco, A. Millán, and I. Ribera. 2014. Tempo and mode of the multiple origins of salinity tolerance in a water beetle lineage. Molecular Ecology 23:360-373.

Badyaev, A. V. 2005. Stress-induced variation in evolution: from behavioural plasticity to genetic assimilation. Proceedings of the Royal Society B 272:877-886.

Bonada, N., and S. Dolédec. 2011. Do mediterranean genera not included in Tachet et al. 2002 have mediterranean trait characteristics? Limnetica 30:129-141.

Bonada, N., S. Dolédec, and B. Statzner. 2007. Taxonomic and biological trait differences of stream macroinvertebrate communities between Mediterranean and temperate regions: implications for future climatic scenarios. Global Change Biology 13:1658-1671.

Bonada, N., and V. Resh. 2013. Mediterranean-climate streams and rivers: geographically separated but ecologically comparable freshwater systems. Hydrobiologia 719:1-29.

Bradley, T. J. 2008. Saline-water insects: ecology, physiology and evolution. Pages 20-35 in J. Lancaster and R. A. Briers, editors. Aquatic insects: challenges to populations. CAB International, Oxfordshire, UK.

Bradshaw, W. E., and C. M. Holzapfel. 2006. Evolutionary response to rapid climate change. Science 312:1477-1478.

Breckels, R. D., and B. D. Neff. 2014. Rapid evolution of sperm length in response to increased temperature in an ectothermic fish. Evolutionary Ecology 28:521-533.

Buchwalter, D. B., D. J. Cain, C. A. Martin, L. Xie, S. N. Luoma, and T. Garland. 2008. Aquatic insect ecophysiological traits reveal phylogenetically based differences in dissolved cadmium susceptibility. Proceedings of the National Academy of Sciences USA 105:8321-8326.

Carew, M. E., V. Pettigrove, R. L. Cox, and A. A. Hoffmann. 2007. The response of Chironomidae to sediment pollution and other environmental characteristics in urban wetlands. Freshwater Biology 52:2444-2462.

Chevenet, F., S. Dolédec, and D. Chessel. 1994. A fuzzy coding approach for the analysis of long-term ecological data. Freshwater Biology 31:295-309.

Clavel, J., R. Julliard, and V. Devictor. 2011. Worldwide decline of specialist species: toward a global functional homogenization? Frontiers in Ecology and the Environment 9:222-228.
Cooper, S. D., P. S. Lake, S. Sabater, J. M. Melack, and J. L. Sabo. 2013. The effects of land use changes on streams and rivers in mediterranean climates. Hydrobiologia 719:383425.

Cothran, R. D., J. M. Brown, and R. A. Relyea. 2013. Proximity to agriculture is correlated with pesticide tolerance: evidence for the evolution of amphibian resistance to modern pesticides. Evolutionary Applications 6:832-841.

Díaz, A. M., M. L. S. Alonso, and M. R. V. A. Gutiérrez. 2008. Biological traits of stream macroinvertebrates from a semiarid catchment: patterns along complex environmental gradients. Freshwater Biology 53:1-21.

Dijkstra, K. D. B., M. T. Monaghan, and S. U. Pauls. 2014. Freshwater biodiversity and aquatic insect diversification. Annual Review of Entomology 59:143-163.

Dolédec, S., J. M. Olivier, and B. Statzner. 2000. Accurate description of the abundance of taxa and their biological traits in stream invertebrate communities: effects of taxonomic and spatial resolution. Archiv für Hydrobiologie 148: 25-43.

Flynn, D. F. B., M. Gogol-Prokurat, T. Nogeire, N. Molinari, B. T. Richers, B. B. Lin, N. Simpson, M. M. Mayfield, and F. DeClerck. 2009. Loss of functional diversity under land use intensification across multiple taxa. Ecology Letters 12:2233.

Garbuz, D. G., O. G. Zatsepina, A. A. Przhiboro, I. Yushenova, I. V. Guzhova, and M. B. Evgen'ev. 2008. Larvae of related Diptera species from thermally contrasting habitats exhibit continuous up-regulation of heat shock proteins and high thermotolerance. Molecular Ecology 17: $4763-4777$.

Gayraud, S., B. Statzner, P. Bady, A. Haybachp, F. Scholl, P. Usseglio-Polatera, and M. Bacchi. 2003. Invertebrate traits for the biomonitoring of large European rivers: an initial assessment of alternative metrics. Freshwater Biology 48: 2045-2064.

Gutiérrez-Cánovas, C., A. Millán, J. Velasco, I. P. Vaughan, and S. J. Ormerod. 2013. Contrasting effects of natural and anthropogenic stressors on beta diversity in river organisms. Global Ecology and Biogeography 22:796-805.

Harrison, S. S. C., and M. Dobson. 2008. Evolutionary drivers and the ecological traits of adult aquatic insects. Pages 250 267 in J. Lancaster and R. A. Briers, editors. Aquatic insects: challenges to populations. CAB International, Wallingford, UK.

Herbst, D. B. 2001. Gradients of salinity stress, environmental stability and water chemistry as a templet for defining habitat types and physiological strategies in inland salt waters. Hydrobiologia 466:209-219.

Higgins, J. K., H. J. MacLean, L. B. Buckley, and J. G. Kingsolver. 2014. Geographic differences and microevolutionary changes in thermal sensitivity of butterfly larvae in response to climate. Functional Ecology 28:982-989.

Hooper, D. U., et al. 2005. Effects of biodiversity on ecosystem functioning: a consensus of current knowledge. Ecological Monographs 75:3-35.

Hopkins, T. L., and K. J. Kramer. 1992. Insect cuticle sclerotization. Annual Review of Entomology 37:273-302.

Jump, A. S., and J. Peñuelas. 2005. Running to stand still: adaptation and the response of plants to rapid climate change. Ecology Letters 8:1010-1020.

Laliberté, E., et al. 2010. Land-use intensification reduces functional redundancy and response diversity in plant communities. Ecology Letters 13:76-86.

Lamouroux, N., S. Dolédec, and S. Gayraud. 2004. Biological traits of stream macroinvertebrate communities: effects of microhabitat, reach, and basin filters. Journal of the North American Benthological Society 23:449-466.

Latta, L. C., L. J. Weider, J. K. Colbourne, and M. E. Pfrender. 2012. The evolution of salinity tolerance in Daphnia: a functional genomics approach. Ecology Letters 15:794-802. 
Luck, G. W., and L. T. Smallbone. 2011. The impact of urbanization on taxonomic and functional similarity among bird communities. Journal of Biogeography 38:894-906.

MacArthur, R., and R. Levins. 1967. Limiting similarity convergence and divergence of coexisting species. American Naturalist 101:377-385.

Maire, V., N. Gross, L. Boerger, R. Proulx, C. Wirth, L. d. S. Pontes, J.-F. Soussana, and F. Louault. 2012. Habitat filtering and niche differentiation jointly explain species relative abundance within grassland communities along fertility and disturbance gradients. New Phytologist 196: 497-509.

Millán, A., J. Velasco, C. Gutiérrez-Cánovas, P. Arribas, F. Picazo, D. Sánchez-Fernández, and P. Abellán. 2011. Mediterranean saline streams in southeast Spain: What do we know? Journal of Arid Environments 75:1352-1359.

Milner, A. M., A. L. Robertson, L. E. Brown, S. H. Sonderland, M. McDermott, and A. J. Veal. 2011. Evolution of a stream ecosystem in recently deglaciated terrain. Ecology 92: 1924-1935.

Monteagudo, L., J. L. Moreno, and F. Picazo. 2012. River eutrophication: irrigated vs. non-irrigated agriculture through different spatial scales. Water Research 46:27592771.

Mouillot, D., N. A. J. Graham, S. Villéger, N. W. H. Mason, and D. R. Bellwood. 2013. A functional approach reveals community responses to disturbances. Trends in Ecology and Evolution 28:167-177.

Odum, E. P. 1985. Trends expected in stressed ecosystems. BioScience 35:419-422.

Parsons, P. A. 2005. Environments and evolution: interactions between stress, resource inadequacy and energetic efficiency. Biological Reviews 80:589-610.

Pfenninger, M., C. Nowak, C. Kley, D. Steinke, and B. Streit. 2007. Utility of DNA taxonomy and barcoding for the inference of larval community structure in morphologically cryptic Chironomus (Diptera) species. Molecular Ecology 16: 1957-1968.

Picazo, F., A. Millán, and S. Dolédec. 2012. Are patterns in the taxonomic, biological and ecological traits of water beetles congruent in Mediterranean ecosystems? Freshwater Biology 57:2192-2210.

Pool, T. K., and J. D. Olden. 2012. Taxonomic and functional homogenization of an endemic desert fish fauna. Diversity and Distributions 18:366-376.

R Development Core Team. 2013. R: a language and environment for statistical computing. R Foundation for Statistical Computing, Vienna, Austria.

Rodrigues, A. R. S., J. B. Torres, H. A. A. Siqueira, and D. P. A. Lacerda. 2013. Inheritance of lambda-cyhalothrin resistance in the predator lady beetle Eriopis connexa (Germar) (Coleoptera: Coccinellidae). Biological Control 64:217-224.

Sala, O. E., et al. 2000. Global biodiversity scenarios for the year 2100. Science 287:1770-1774.

Salminen, J., C. A. M. van Gestel, and J. Oksanen. 2001. Pollution-induced community tolerance and functional re- dundancy in a decomposer food web in metal-stressed soil. Environmental Toxicology and Chemistry 20:2287-2295.

Sánchez-Fernández, D., P. Abellán, A. Mellado, J. Velasco, and A. Millán. 2006. Are water beetles good indicators of biodiversity in Mediterranean aquatic ecosystems? The case of the Segura River Basin (SE Spain). Biodiversity and Conservation 15:4507-4520.

Scanlon, B. R., I. Jolly, M. Sophocleous, and L. Zhang. 2007. Global impacts of conversions from natural to agricultural ecosystems on water resources: quantity versus quality. Water Resources Research 43.

Schmera, D., B. Baur, and T. Eros. 2012. Does functional redundancy of communities provide insurance against human disturbances? An analysis using regional-scale stream invertebrate data. Hydrobiologia 693:183-194.

Scoville, A. G., and M. E. Pfrender. 2010. Phenotypic plasticity facilitates recurrent rapid adaptation to introduced predators. Proceedings of the National Academy of Sciences USA 107:4260-4263.

Shipley, B., D. Vile, and E. Garnier. 2006. From plant traits to plant communities: a statistical mechanistic approach to biodiversity. Science 314:812-814.

Southwood, T. R. E. 1977. Habitat, templet for ecological strategies? Presidential address to British Ecological Society, 5 January 1977. Journal of Animal Ecology 46:337-365.

Stanton, M. L., B. A. Roy, and D. A. Thiede. 2000. Evolution in stressful environments. I. Phenotypic variability, phenotypic selection, and response to selection in five distinct environmental stresses. Evolution 54:93-111.

Statzner, B., N. Bonada, and S. Dolédec. 2007. Conservation of taxonomic and biological trait diversity of European stream macroinvertebrate communities: a case for a collective public database. Biodiversity and Conservation 16:3609-3632.

Stubbs, W. J., and J. B. Wilson. 2004. Evidence for limiting similarity in a sand dune community. Journal of Ecology 92: $557-567$.

Tachet, H., P. Richoux, M. Bournaud, and P. UsseglioPolatera. 2002. Invertébrés d'eau douce. Systematique, biologie, écologie (2nd corrected impression). CNRS éditions, Paris, France.

Ulrich, W., and N. J. Gotelli. 2007. Null model analysis of species nestedness patterns. Ecology 88:1824-1831.

Villéger, S., N. W. H. Mason, and D. Mouillot. 2008. New multidimensional functional diversity indices for a multifaceted framework in functional ecology. Ecology 89:22902301.

Villéger, S., P. M. Novack-Gottshall, and D. Mouillot. 2011. The multidimensionality of the niche reveals functional diversity changes in benthic marine biotas across geological time. Ecology Letters 14:561-568.

Violle, C., B. J. Enquist, B. J. McGill, L. Jiang, C. H. Albert, C. Hulshof, V. Jung, and J. Messier. 2012. The return of the variance: intraspecific variability in community ecology. Trends in Ecology and Evolution 27:244-252.

Weiher, E., D. Freund, T. Bunton, A. Stefanski, T. Lee, and S. Bentivenga. 2011. Advances, challenges and a developing synthesis of ecological community assembly theory. Philosophical Transactions of the Royal Society B 366:2403-2413.

\section{Supplemental Material}

\section{Ecological Archives}

Appendices A-D and the Supplement are available online: http://dx.doi.org/10.1890/14-1447.1.sm 